\title{
The role of primary cilia in the molecular pathogenesis of phaeochromocytoma
}

\author{
Sam M. O'Toole 1, 2, Umasuthan Srirangalingam¹, 2, William M. Drake1, 2 \& J. Paul Chapple ${ }^{1}$ \\ ${ }^{1}$ Centre for Endocrinology, William Harvey Research Institute, Barts and the London School of Medicine and \\ Dentistry, Queen Mary University of London, London, UK \\ ${ }^{2}$ Department of Endocrinology, St Bartholomew's Hospital, Barts Health NHS Trust, London, UK
}

\begin{abstract}
1. Introduction
Phaeochromocytomas are neuroendocrine tumours arising from adrenal medulla chromaffin cells. They are life threatening due to adrenaline and noradrenaline release and potential for metastatic spread. Understanding of phaeochromocytoma pathogenesis is incomplete with limited ability to predict malignant potential. Additionally, once metastatic, response to conventional therapies is disappointing.

Phaeochromocytomas are a common feature of the inherited cancer syndrome von Hippel-Lindau disease, which is caused by loss of function of the VHL protein. As well as its canonical function in degradation of the transcription factor hypoxiainducible factor, VHL is implicated in formation and maintenance of primary cilia. These are organelles that protrude from the cells, functioning in transduction of extracellular signals. This is dependent on localisation of signalling components to cilia, including proteins linked to pathways that are dysregulated in tumorigenesis. Moreover cilia are believed to act as a checkpoint for cell division because they assemble from the basal body, which is a modified centriole and thus required for spindle pole formation at the end of interphase.

In this study we test the hypothesis that primary cilia structure is disrupted in phaeochromocytomas
\end{abstract}

2. What are primary cilia?

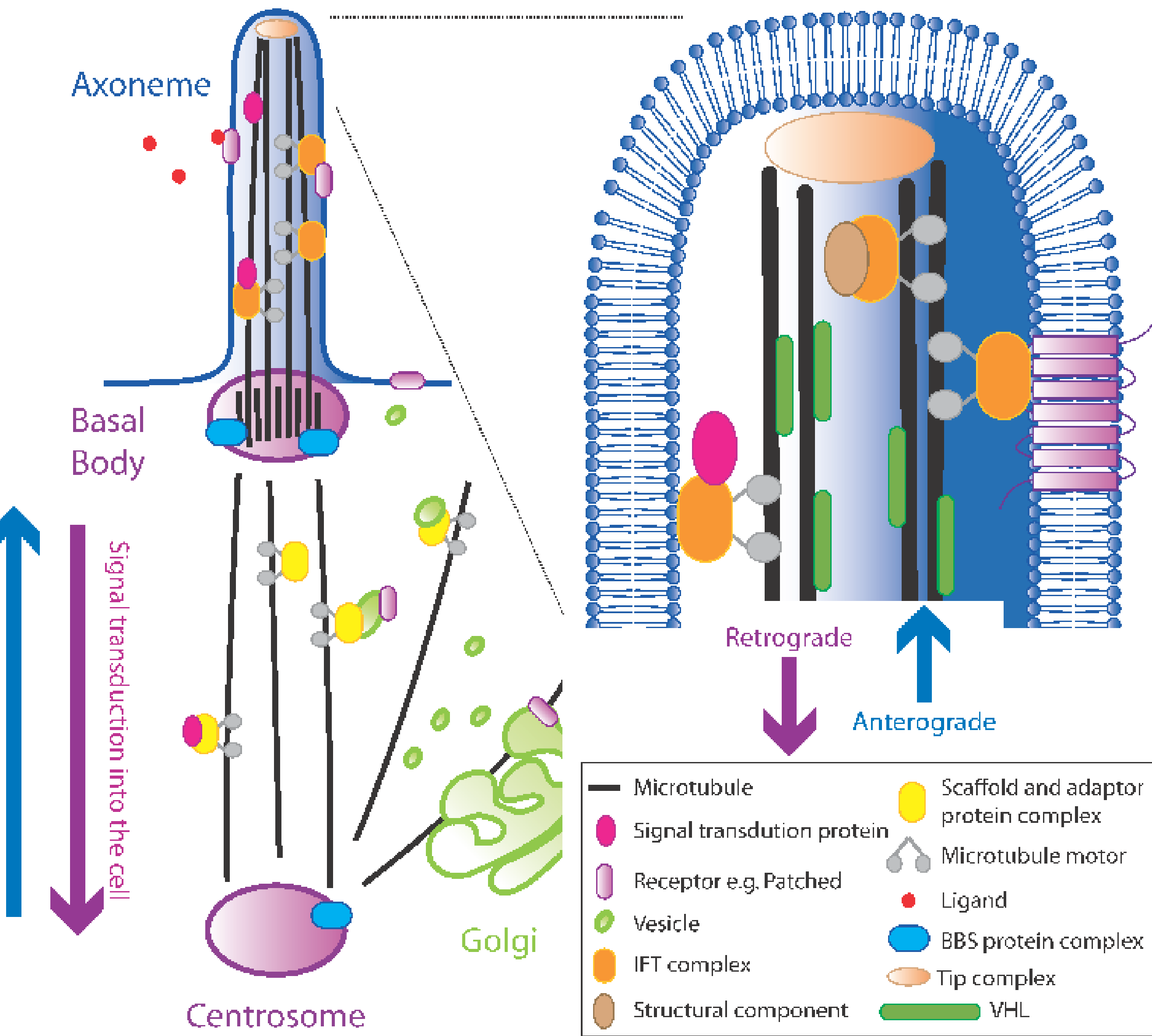

Structure: Primary cilia consist of a microtubule-based core (the axoneme), which elongates from a basal body, and is covered by the ciliary membrane. Cilia formation and maintenance is sensitive to the cellular microenvironment. Cell cycle: Primary cilia are dynamic structures that elongate during stationary phase, with resorption occurring when the basal body is required to form the centriole during mitosis Signalling: Primary cilia are signalling platforms involved in the transduction of a range of extracellular stimuli. Cilia-mediated signalling pathway (e.g. hedgehog, Wnt and PDGFR $\alpha$ ) are regulated by the control and compartmentalisation of pathway components within the axoneme and ciliary membrane Cancer: Primary cilia are lost in a range of cancers. This includes clear cell renal cancer, which is associated with von Hippel-Lindau disease. A functions of the VHL protein is to maintain the primary cilium by stabilising microtubules.

\section{Phaeochromocytoma and pseudohypoxia} Pseudohypoxia (HIF activation in the presence of oxygen) is a cardinal feature of Cluster 1 phaeochromocytomas

Mutations in VHL and succinate dehydrogenase (SDH) result in pseudohypoxia and phaeochromocytoma development VHL targets HIF for degradation by the proteasome mutations prevent this, resulting in HIF accumulation

SDH converts succinate to fumarate - mutations result in succinate accumulation thereby inhibiting prolyl hydroxylases and preventing $\mathrm{VHL}$ binding and destroying $\mathrm{HIF}$
A. Immunofluorescent detection of primary cilia in cryo-sectioned phaeochromocytoma and normal adjacent tissue. Scale bar $=10$

http://www.primarycilia.qmul.ac.uk

http://www.whri.qmul.ac.uk/staff-all/176-chapple-pau
4. Primary cilia incidence and length is reduced in phaeochromocytoma
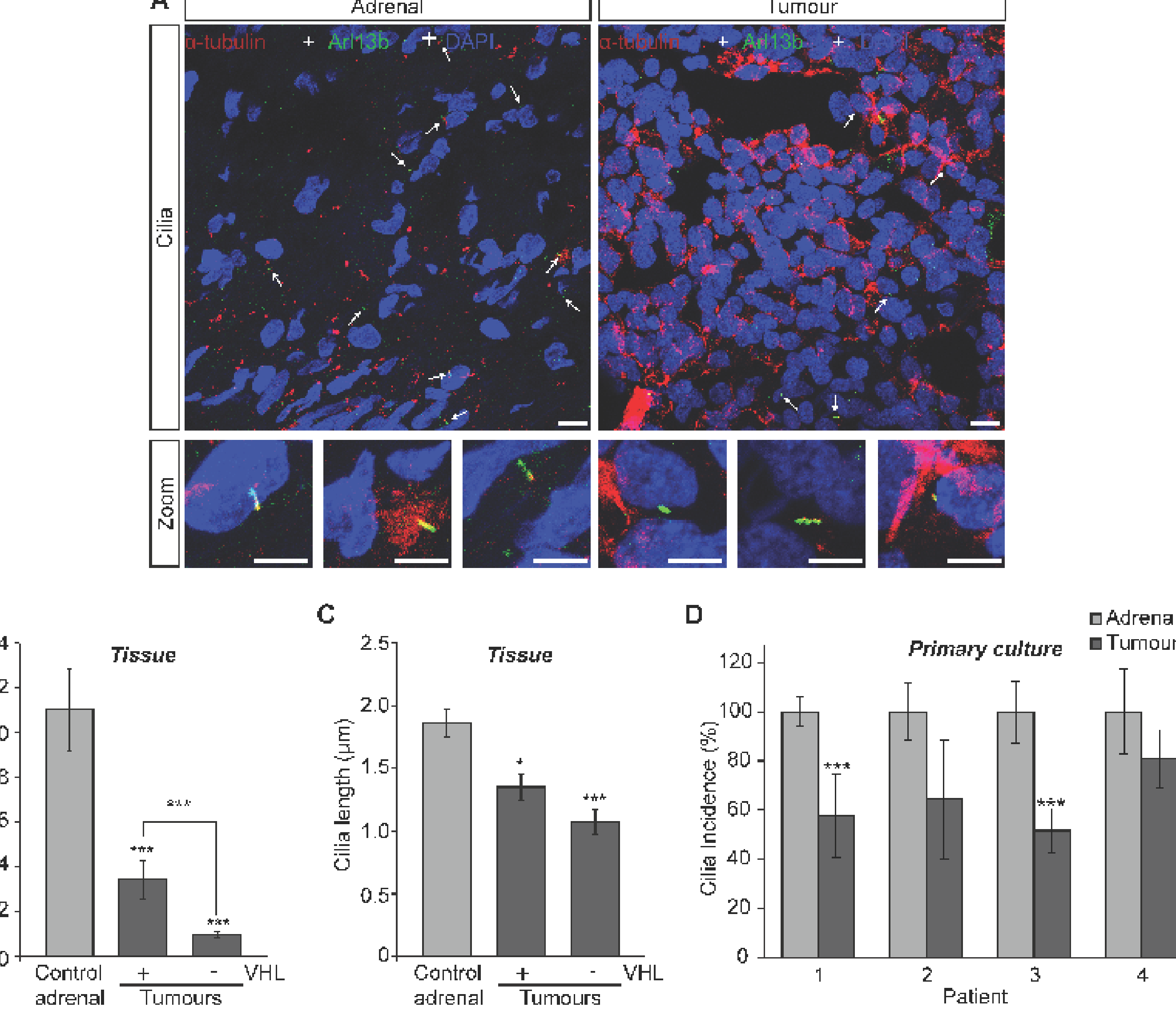
$\mu \mathrm{m}$

B. Quantification of cilia incidence in phaeochromocytoma and normal adjacent tissue.

C. Quantification of cilia length in phaeochromocytoma and normal adjacent tissue.

tissution of cilia incidence in primary cultures from phaeochromocytoma and normal adjacent tissue in four patients.

5. Knockdown of the essential cilia protein IFT88 results in cilia loss and increased cellular proliferation in the

phaeochromocytoma PC12 cell line

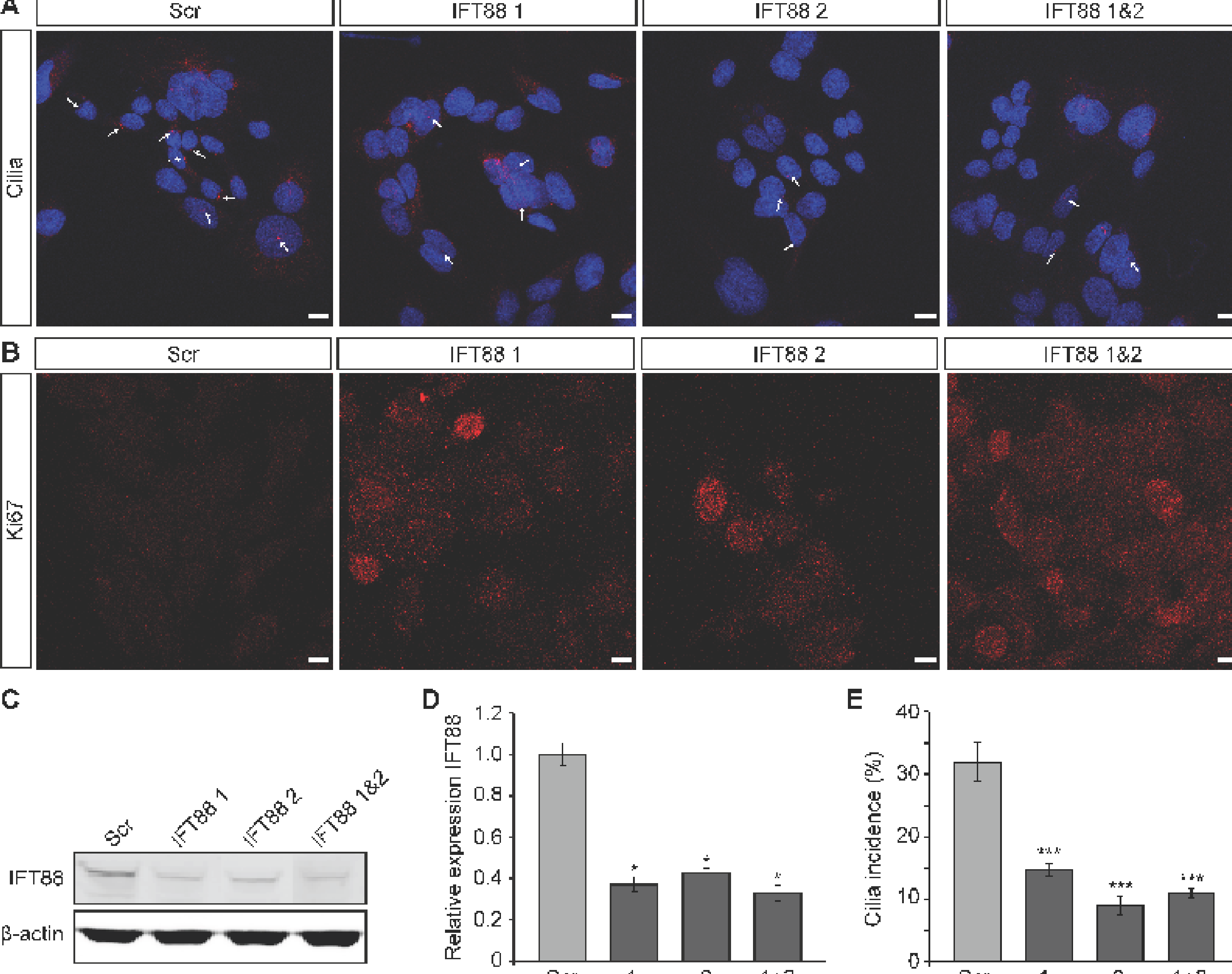

6. PC12 primary cilia are sensitive to hypoxia

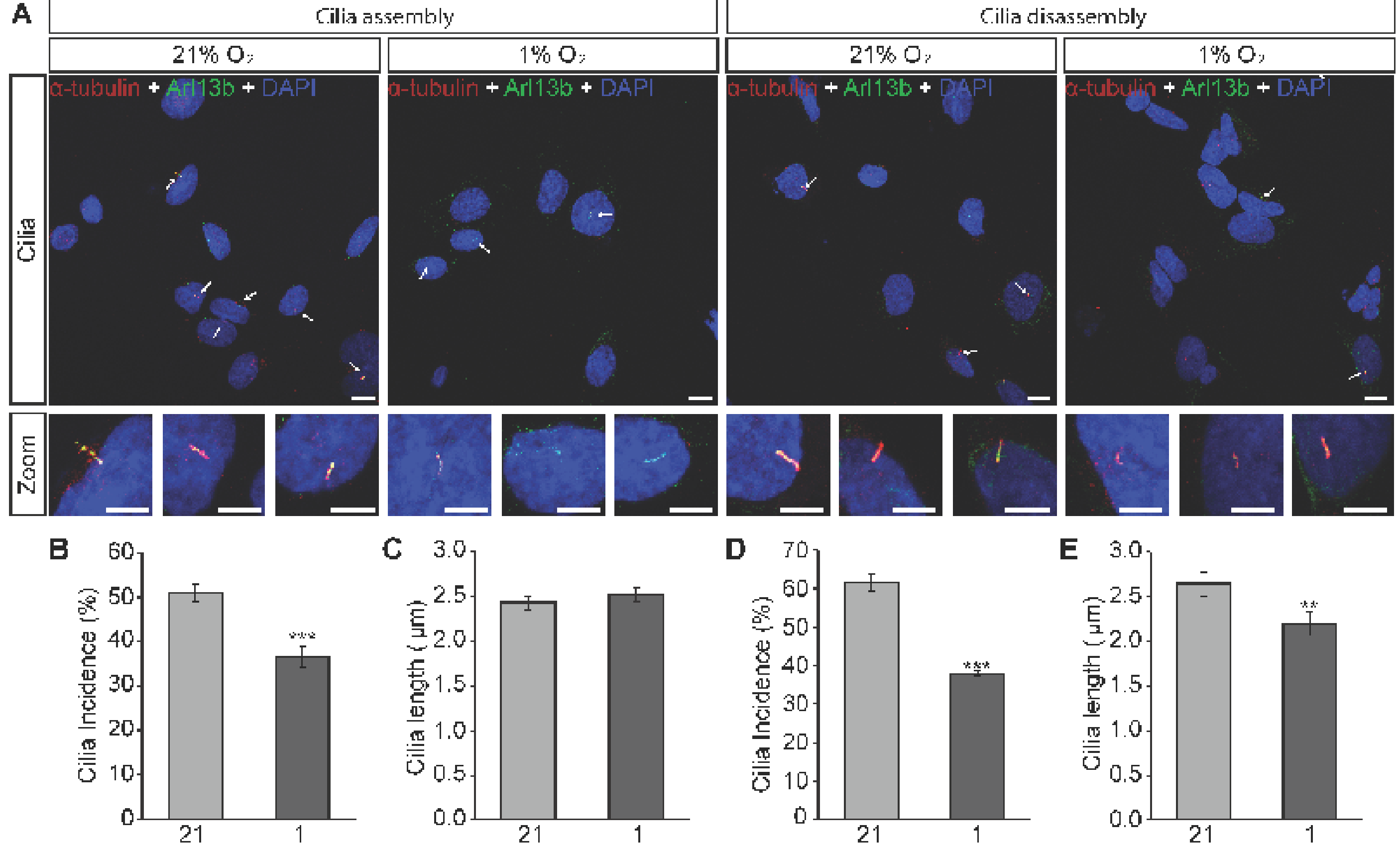

A. Immunofluorescent detection of primary cilia in PC12 cells transferred to hypoxic conditions before and after ciliogenesis. B \& C. Quantification of cilia assembly in PC12 cells exposed to low

$D$ \& E . Quantification of cilia disassembly in PC12 cells exposed to low oxygen conditions.

7. Induction of pseudohypoxia by targeting SDHB results in primary cilia loss and increased PC12 proliferation
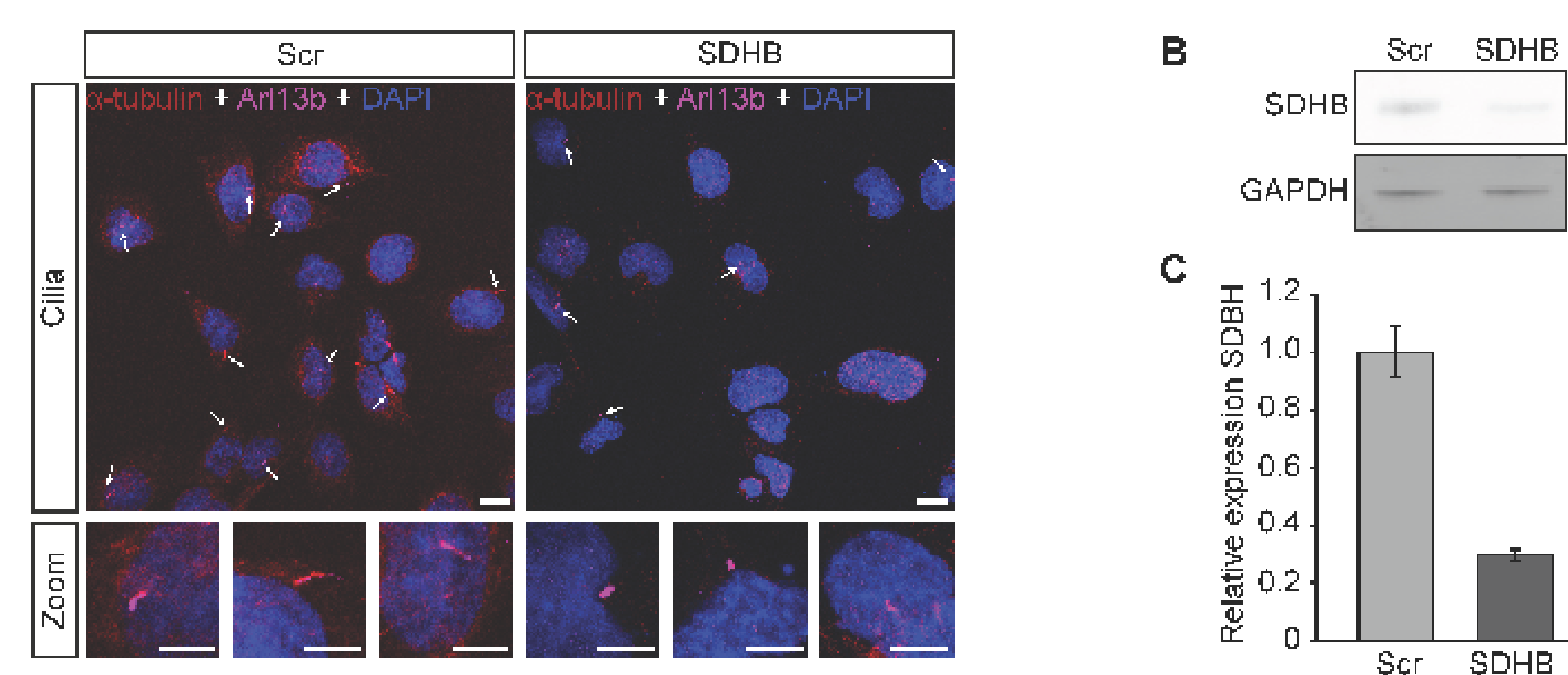

Scr SDHB
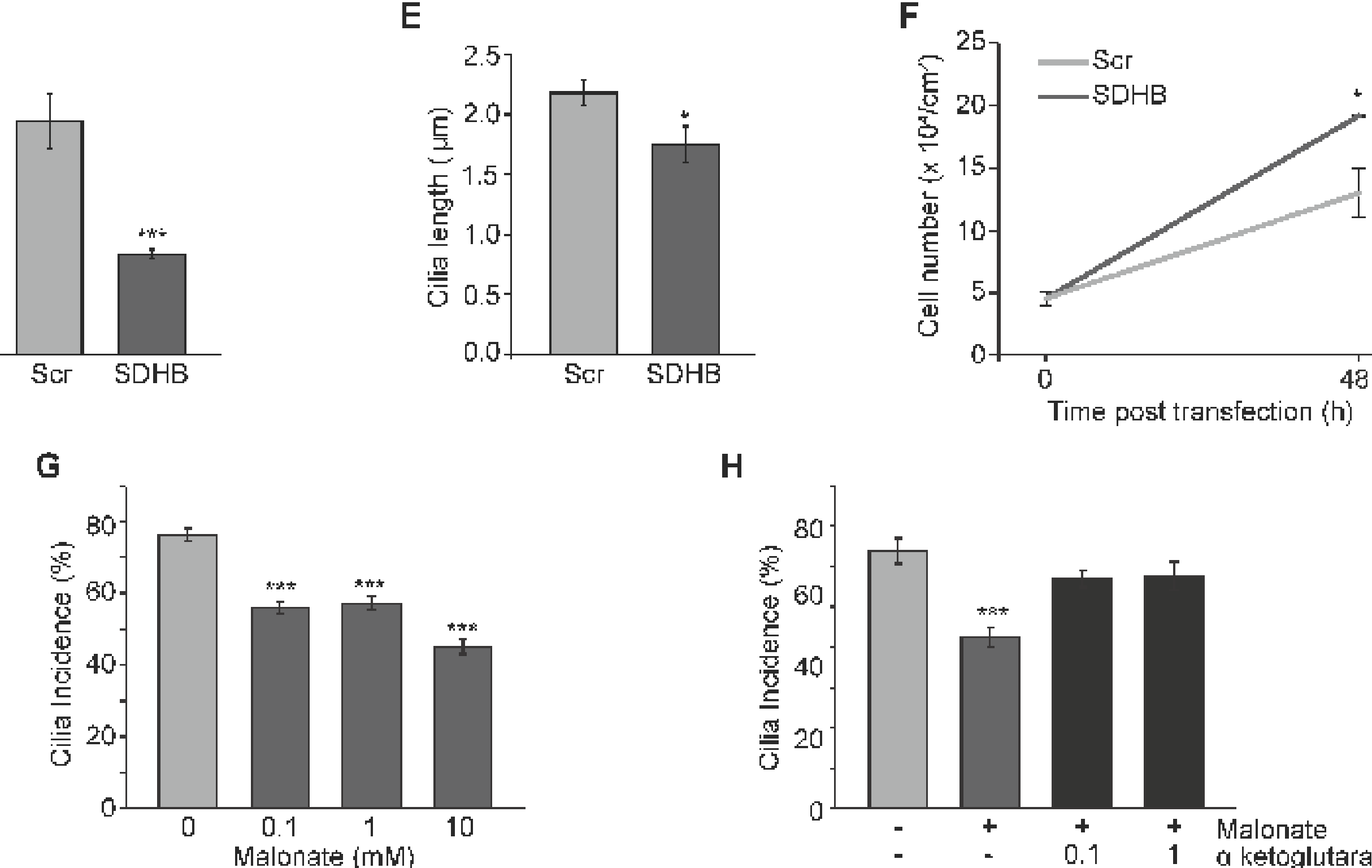

A. Immunofluorescent detection of primary cilia in PC12 cells A. Insfected with siRNAs targeting SDHB.

$B$ \& C. Immunoblot and quantification of siRNA-mediated SDHB knockdown

D. Quantification of cilia incidence in SDHB knockdown PC12 cells E. Quantification of cilia length in SDHB knockdown PC12 cells. F. Quantification of cell number 48 hours after transfection with siRNAs targeting SDHB.

Q Quantification of cilia incidence in PC12 cells treated with the $\mathrm{SDH}$ inhibitor malonate, which causes succinate to accumulate. $H$. Quantification of cilia incidence in PC12 cells treated with the SDH inhibitor malonate and a ketoglutarate which opposes the inhibitory action of succinate on HIF prolyl hydroxylases.

8. Conclusions

A. Immunofluorescent detection of primary cilia in PC12 cells A. Imsfected with siRNAs targeting IFT88.

B. Ki67 staining of PC12 cells transfected with siRNAs targeting

C \& D. Immunoblot and quantification of siRNA-mediated IFT88 knockdown

E. Quantification of cilia incidence in IFT88 knockdown cells. F. Quantification of cell number 48 hours after transfection with siRNAs targeting IFT88.

G. Quantification of cell proliferation by Ki67 staining
- Primary cilia loss is a feature of phaeochromocytomas

Primary cilia loss in PC12 cells results in increased cellular proliferation

Primary cilia in PC12 cells are sensitive to hypoxia and increased cellular proliferation
Loss of SDH function in PC12 cells results in cilia loss 